\title{
Application of Structural Deformation Monitoring Based on Close-Range Photogrammetry Technology
}

\author{
Jun Hu, ${ }^{1,2}$ Ensheng Liu $\mathbb{D}^{3,4}$ and Jiayu Yu ${ }^{1,2}$ \\ ${ }^{1}$ State Key Laboratory of Mountain Bridge and Tunnel Engineering, Chongqing Jiaotong University, Chongqing 400074, China \\ ${ }^{2}$ School of Civil Engineering, Chongqing Jiaotong University, Chongqing 400074, China \\ ${ }^{3}$ College of Building Engineering, Jinggangshan University, Jian 343009, Jiangxi, China \\ ${ }^{4}$ College of Surveying and Geo-Informatics, Tongji University, Shanghai 200092, China \\ Correspondence should be addressed to Ensheng Liu; 1410893@tongji.edu.cn
}

Received 8 December 2020; Revised 5 January 2021; Accepted 20 January 2021; Published 20 February 2021

Academic Editor: Sang-Bing Tsai

Copyright ( 2021 Jun Hu et al. This is an open access article distributed under the Creative Commons Attribution License, which permits unrestricted use, distribution, and reproduction in any medium, provided the original work is properly cited.

\begin{abstract}
The problem of structural deformation monitoring has always been a research hotspot in engineering architecture. Research on structural deformation monitoring is the main way to solve the risk of engineering construction. Based on using closerange photogrammetry technology to monitor structural deformation, combining with image recognition technology to collect and process image data, and using the conditions of direct linear transformation and collinear equation to calculate based on the extracted coordinates of the measuring point, finally, according to the coordinates of the measuring point and the deflection value of the bridge structure, the study is carried out. Experiment: first, five sets of data are used to calibrate the close-range photogrammetry equipment of measurement point accuracy are analyzed, then the bridge piers and the measurement point coordinates, the lasso, under different load on the accuracy of close-range photogrammetry technology is analyzed, according to the relevant data of the error of the close-range photogrammetry technology are analyzed, and through the calculation of different measurement methods to analyze the direct linear transformation manifested in the article. The final results show that the $X$-axis coordinate range of the measured points in the auxiliary spatial coordinate system is $25 \sim 40, Y$-axis coordinate range is $8 \sim 36$, and $Z$-axis coordinate range is $10 \sim 35$. The coordinates of measuring points of bridge cables are distributed in the fourth quadrant of the spatial coordinate system. The accuracy values of all coordinates of the measuring points under the close-range photogrammetry equipment are less than $0.1 \mathrm{~mm}$, indicating that the closerange photogrammetry equipment has a good effect on the calibration of measuring points, and the error generated when the load action of each measuring point of the bridge cable is $10 \mathrm{~N}$ is greater than that generated when the load action is $20 \mathrm{~N}$. In different calculation and measurement methods, the results obtained by total station measurement and calculation method are reduced by about $0.04 \sim 0.07 \mathrm{~m}$ compared with the actual value; the results obtained by direct linear transformation method are increased by about $0.02 \sim 0.04 \mathrm{~m}$ compared with the actual value; and the results obtained by other measurement and calculation methods are increased by about $0.04 \sim 0.06 \mathrm{~m}$ compared with the actual value.
\end{abstract}

\section{Introduction}

1.1. Background Meaning. In recent years, China's economy has developed rapidly, and there is an increasing demand for building construction [1], which is accompanied by quality risks brought by various buildings and threats to people's life and property safety. All buildings have a certain service life. If the building is used for a long time, it needs to be repaired regularly; otherwise, the interior and main body of the building will have structural deformation and other problems, which can easily cause the building to break and collapse, posing a huge threat to people's life, health, and property safety The deformation of the building is mainly caused by rain erosion, air oxidation, and aging of the stressed parts under long-term load, which lead to the reduction of the strength and 
stiffness of the building. As a very important part of traffic engineering, bridge deformation has been concerned by many architects and people from all walks of life.

Close-range photogrammetry technology is a very important measurement method in the research of building deformation monitoring. Close-range photogrammetry technology can accurately record the information of the measured object in an instant and obtain the point position relationship of the measured object; and this technology is very practical to measure objects of any shape and can also measure the information of dynamic objects, and it can also help people measure dangerous objects. The image information obtained in the close-range photogrammetry technology can be stored for a long time, which is convenient for the analysis of subsequent experiments.

1.2. Related Work. At present, many scholars have carried out relevant research on the application of structural deformation monitoring. Kim used close-range photogrammetry to study the main reflecting surface monitoring system based on high-efficiency algorithms, in order to become a continuous automatic monitoring system for structural deformation in the near future. Kim used TLS to estimate 10 fitting lines on the characteristic points distributed on the main reflecting surface. On the basis of nonintersecting straight lines, the nearest point algorithm was used to calculate the composite intersection point of the fitting lines, and the calculation was based on the intuitive basis of time series analysis. The result can provide the numerical change at the intersection point represented by the three axes, opening the way for predicting the deformation rate and direction [2]. Chen proposed a spatiotemporal clustering and health diagnosis method for ultrahigh concrete arch DAMS based on the monitoring data of vertical meter [3]. First, he proposed a spatiotemporal expression method for deformation monitoring data, which upgraded a time series with punctuation to a time series of curved surface with cross section, dam axis, and time change direction; second, on the basis of deeply discussing the similarity characteristics of deformation space and time, the comprehensive similarity indexes of absolute distance, incremental distance, and growth rate distance are constructed; third, on the basis of preserving key features (i.e., extremum point and turning point), a time clustering method is proposed by eliminating irrelevant details (i.e., noise point); and finally, a multiscale fuzzy C-means data mining method and its iterative algorithm are designed to realize the temporal and spatial clustering of dam deformation [4]. Lopato introduced the application of microstrip antenna sensors in the deformation monitoring of bent steel structures. This sensor element can be used in a structural health monitoring system, using patch sensors to measure deformation. For samples in the bending process, the sensor geometry becomes nonplanar. This fact has an impact on the measured resonance frequency, so it needs to be studied. In order to analyze the influence of the curvature of the patch sensor on the resonance frequency during the bending process, Lopato conducted a finite element simulation, and the analysis results were verified by experimentation [5]. Sun reviewed the research and development history of aircraft structural deformation monitoring; analyzed the main technical types, advantages and disadvantages, on-board applicability, deformation reconstruction algorithms, and typical application examples of optical fiber monitoring technology; pointed out the key issues and main application modes; and updated materials, standardization, intelligence, and other aspects to explore the future direction of development [6]. Zhang used a distributed long-diameter optical fiber sensor to test and analyze the environmental vibration of a large-span rigid frame bridge [7]. He proposed the concept of a long-diameter optical fiber sensor and its advantages in revealing local and overall structural features. The long-span bridge was monitored, and a method for calculating the structural deformation distribution from the measured long strain was proposed. The measured longgauge dynamic strain was used to identify the modal of the bridge under study, and the strain and displacement modes were identified from it [8]. Although the above methods have achieved good results, there are still some defects. Kim's and Chen's methods require high experimental costs, Lopato's method is not comprehensive enough, and Sun's and Zhang's methods require too much data.

1.3. Innovation in This Article. This paper uses close-range photogrammetry technology to capture the relevant data of the bridge, which can obtain high-precision bridge images and can obtain the information of multiple measurement points of the bridge at the same time, which reduces the time of experimental data collection. The close-range photogrammetry technology does not need to directly contact with the measured object, which guarantees the natural state of the object. The experiment uses direct linear conversion and collinear condition equations to calculate the data, which improves the calculation efficiency of the experimental data. This research uses image recognition technology to process the images obtained in the experiment and calibrate the measurement points while enhancing the image.

\section{Related Technologies for Structural Deformation Monitoring}

2.1. Close-Range Photogrammetry. Close-range photogrammetry is a branch of photogrammetry and remote sensing technology. Close-range photogrammetry can obtain relevant information of the Earth and other objects in the surrounding environment from noncontact imaging and other sensing systems through recording, measurement, analysis, and expression within the range of photography distances less than $100 \mathrm{~m}[9,10]$. Close-range photogrammetry is also a photogrammetry of the shape and movement of various objects [11]. This technology has been widely used in various research studies in the fields of topography, industry, biomedicine, and architecture. Close-range photogrammetry technology has become more and more perfect and mature after decades of development and research, and it can meet the requirements of image accuracy and 
precision in various fields [12]. At present, the research focus of close-range photogrammetry technology has changed from geometric measurement accuracy to automation and 3D modeling based on virtual reality technology.

Compared with other three-dimensional measurement technologies, the close-range photogrammetry technology has real-time performance in obtaining relevant information of the measured object and can also measure objects with more measurement points. Proximity photogrammetry technology is a noncontact measurement method. It does not need to directly contact the object to be measured. Therefore, it has no higher requirements on the measurement environment and the measurement object and can adapt to harsh environments $[13,14]$. Close-range photogrammetry can not only measure static objects but also measure the shape and motion state of dynamic objects. It plays an important role in the measurement of the microscopic world and distant objects [15]. With the continuous advancement of technology, close-range photogrammetry technology can provide users with high-precision reliability measurements according to different processing methods and capital investment. Close-range photogrammetry technology can not only measure the three-dimensional coordinates of the target point but also measure characteristic quantities such as amplitude and low-speed motion frequency [16].

Although the performance of close-range photogrammetry measurement accuracy is good, it is not necessarily the best technology choice for all measurement objects. Researchers need to select appropriate measurement methods according to all aspects of the experiment. In addition, the technical content of close-range photogrammetry is relatively high, and the requirements for hardware equipment and technical personnel are also relatively high. If the hardware equipment has defects or the technology does not meet the requirements, it will cause errors in the measurement results and affect the final results of the experiment.

2.2. Deformation Monitoring. Deformation monitoring refers to the long-term observation of the deformation phenomenon of deformed objects through special measuring equipment and technology and the analysis and prediction of the deformation form and development trend of deformed objects $[17,18]$. The measured objects of deformation monitoring can be the whole Earth, or a certain region, or a building. According to the measured objects, deformation monitoring can be divided into global deformation monitoring, regional deformation monitoring, and engineering deformation monitoring $[19,20]$. The measurement precision of deformation monitoring is very high, and it belongs to precision measurement [21]. In the monitoring of the structural deformation of the building, by observing whether the structure of the building is deformed or not, the abnormal situation of the building can be discovered and dealt with in time, thereby ensuring the safety of the engineering personnel and the building. Using deformation monitoring to analyze the deformation of the building, the construction and design and rationality of the building can be tested and the quality of the project can be detected, which is of great significance to the prediction and prevention of future accidents of the construction project.

In deformation monitoring, direct linear transformation can be used to calculate and analyze the object information in the close-range film and television measurement coordinate system [22]. Using the direct linear change method, find the coordinates in the space above the image coordinates, calculate, and analyze the direct linear relationship.

$$
\lambda\left[\begin{array}{lll}
x & y & 1
\end{array}\right]^{T}=G\left[\begin{array}{lll}
X & Y & 1
\end{array}\right]^{T} .
$$

The left side of formula (1) represents the pixel coordinates, the right side represents the object point coordinates, $\lambda$ is the scale factor between them, and $G$ is the perspective matrix:

$$
G=\left[\begin{array}{lll}
g_{11} & g_{12} & g_{13} \\
g_{21} & g_{22} & g_{23} \\
g_{31} & g_{32} & 1
\end{array}\right] .
$$

Substituting formula (2) into formula (1) and eliminating the scale factor, two linear equations can be obtained:

$$
\left\{\begin{array}{l}
g_{11} X+g_{12} Y+g_{13}-x g_{31} X-y g_{32} Y=x, \\
g_{21} X+g_{22} Y+g_{23}-y g_{31} X-y g_{32} Y=y .
\end{array}\right.
$$

2.3. Image Identification. Image recognition refers to a technology of feature extraction and classification recognition of collected image data through computer equipment [23]. The traditional image recognition process is divided into image acquisition, image preprocessing, feature extraction, and image recognition. The related problem of image recognition is the mapping from the simulation space to the category space, which needs to take the main features of the image as the basis to extract the relevant information of the effective features [24-26]. The image data collected in close-range photogrammetry are digital images. Digital image processing and recognition started at a very early time. A digital image has more advantages than an analog image, which are mainly reflected in the storage, transportation, and processing of data. These advantages provide a strong power for image recognition technology. After continuous improvement and development, the three main recognition methods of image recognition technology are statistical pattern recognition, structural pattern recognition, and fuzzy pattern recognition [27].

In the process of image recognition and processing, image segmentation is an indispensable key technology. It divides the tested image into several parts according to specific attributes through some technical means, which is a key step from image processing to image analysis [28]. There are many existing image segmentation methods, including segmentation methods based on image features and segmentation methods based on specific theoretical tools [29]. 
Among many image segmentation methods, gray threshold segmentation is the most commonly used method. This kind of method mainly determines an appropriate threshold value, then compares the threshold value with the gray value of the image, and divides the image according to the threshold value $[30,31]$. Image recognition plays an important role in computer vision, artificial intelligence, and other fields and is widely used in motion recognition, face recognition, image processing, and other related research [32].

\section{Structural Deformation Monitoring Experiment Design}

3.1. Data Collection. For study of deformation monitoring structures, first, it is necessary to get a digital image data of the measured object. Digital image acquisition object has to be measured by the need for a spectral band of electromagnetic energy-sensitive element and capable of physicalelectrical signal into a digital signal digitized devices [33]. These image acquisition devices can receive external stimuli and generate responses. The physical components can receive radiation and generate electrical signals proportional to electromagnetic radiation and then convert the electrical signals into digital signals through digital devices and transmit them to the computer.

\subsection{Application of Collinear Condition Equation in Structural} Deformation Monitoring. There are three commonly used coordinate systems in close-range photogrammetry, which are the image plane coordinate system $O-x y$, the image space coordinate system $S-x y z$, and the object space coordinate system $O-X Y Z$. The collinear condition equation in deformation monitoring is a condition equation describing that the image point, photography center, and object direction point should be on the same straight line. It is the most important analytical basis in photogrammetry. There is a point $A$ in the space coordinate system $S-x y z$ whose coordinate is $S-x y z$, and the coordinate $(X, Y, Z)$ of this point in the auxiliary space coordinate system $S-X Y Z$ needs to be solved. It is known that the transformation from a spatial coordinate system to an auxiliary spatial coordinate system requires the sequential rotation of three angles $(\alpha \longrightarrow \beta \longrightarrow \theta)$, all of which are defined as positive values. The relationship between the point in the coordinate system and the spatial coordinate system $S-x_{\theta} y_{\theta} z_{\theta}$ can be expressed as

$$
\left[\begin{array}{l}
x_{\theta} \\
y_{\theta} \\
z_{\theta}
\end{array}\right]=\left[\begin{array}{ccc}
\cos \theta & -\sin \theta & 0 \\
\sin \theta & \cos \theta & 0 \\
0 & 0 & 1
\end{array}\right]\left[\begin{array}{c}
x \\
y \\
-e
\end{array}\right]=R_{\theta}\left[\begin{array}{c}
x \\
y \\
-e
\end{array}\right] .
$$

The value of this point $z_{\theta}$ is the same as $z$ in these two coordinate systems. The coordinate relationship between the point in the coordinate system $S-x_{\theta \beta} y_{\theta \beta} z_{\theta \beta}$ and the coordinate system $S-x_{\theta} y_{\theta} z_{\theta}$ can be expressed as

$$
\left[\begin{array}{l}
x_{\theta \beta} \\
y_{\theta \beta} \\
z_{\theta \beta}
\end{array}\right]=\left[\begin{array}{ccc}
1 & 0 & 0 \\
0 & \cos \beta & -\sin \beta \\
0 & \sin \beta & \cos \beta
\end{array}\right]\left[\begin{array}{l}
x_{\theta} \\
y_{\theta} \\
z_{\theta}
\end{array}\right]=R_{\beta}\left[\begin{array}{l}
x_{\theta} \\
y_{\theta} \\
z_{\theta}
\end{array}\right] .
$$

The value of this point $x_{\theta \beta}$ is the same as the value of $x_{\theta}$ in these two coordinate systems. The coordinate relationship between the point in the auxiliary space coordinate system and the coordinate system $S-x_{\theta \beta} y_{\theta \beta} z_{\theta \beta}$ can be expressed as

$$
\left[\begin{array}{l}
X \\
Y \\
Z
\end{array}\right]=\left[\begin{array}{ccc}
\cos \alpha & 0 & -\sin \alpha \\
0 & 1 & 0 \\
\sin \alpha & 0 & \cos \alpha
\end{array}\right]\left[\begin{array}{l}
x_{\theta \beta} \\
y_{\theta \beta} \\
z_{\theta \beta}
\end{array}\right]=R_{\alpha}\left[\begin{array}{c}
x_{\theta \beta} \\
y_{\theta \beta} \\
z_{\theta \beta}
\end{array}\right] .
$$

The value of $Y$ at this point is the same as the value of $y_{\theta \beta}$ in these two coordinate systems. Substitute formula (4) into formula (5), and finally merge into formula (6) to get the coordinates of the point in the auxiliary space coordinate system:

$$
\left[\begin{array}{c}
X \\
Y \\
Z
\end{array}\right]=R_{\alpha} R_{\beta} R_{\theta}\left[\begin{array}{c}
x \\
y \\
-e
\end{array}\right]=\left[\begin{array}{lll}
a_{0} & a_{1} & a_{2} \\
b_{0} & b_{1} & b_{2} \\
c_{0} & c_{1} & c_{2}
\end{array}\right]\left[\begin{array}{c}
x \\
y \\
-e
\end{array}\right] .
$$

In formula (7),

$$
\begin{aligned}
& a_{0}=\cos \alpha \cos \theta-\sin \alpha \sin \beta \sin \theta, \\
& a_{1}=-\cos \alpha \sin \theta-\sin \alpha \sin \beta \sin \theta \\
& a_{2}=-\sin \alpha \cos \beta \\
& b_{0}=\cos \beta \sin \theta \\
& b_{1}=\cos \beta \cos \theta \\
& b_{2}=-\sin \beta \\
& c_{0}=\sin \alpha \cos \theta+\cos \alpha \cos \beta \cos \theta, \\
& c_{1}=-\sin \alpha \sin \theta+\cos \alpha \sin \beta \cos \theta, \\
& c_{2}=\cos \alpha \cos \beta
\end{aligned}
$$

Since $R$ is an orthogonal matrix, for formula (7),

$$
\left[\begin{array}{c}
x \\
y \\
-e
\end{array}\right]=R^{-1}\left[\begin{array}{c}
X \\
Y \\
Z
\end{array}\right]=\left[\begin{array}{lll}
a_{0} & b_{0} & c_{0} \\
a_{1} & b_{1} & c_{1} \\
a_{2} & b_{2} & c_{2}
\end{array}\right]\left[\begin{array}{l}
X \\
Y \\
Z
\end{array}\right]
$$

Suppose $\left(X_{s}, Y_{s}, Z_{s}\right)$ is the object space coordinate of the shooting point, $\left(X_{0}, Y_{0}, Z_{0}\right)$ is the object point space coordinate, $k$ is the scaling factor between the coordinate and $\left(X_{0}-X_{s}, Y_{0}-Y_{s}, Z_{0}-Z_{s}\right)$ in the auxiliary space coordinate system, and $e$ is the internal orientation element of the image, we can get 


$$
\left[\begin{array}{c}
x \\
y \\
-e
\end{array}\right]=k^{-1}\left[\begin{array}{c}
X \\
Y \\
Z
\end{array}\right]=\left[\begin{array}{lll}
a_{0} & b_{0} & c_{0} \\
a_{1} & b_{1} & c_{1} \\
a_{2} & b_{2} & c_{2}
\end{array}\right]\left[\begin{array}{c}
X-X_{s} \\
Y-Y_{s} \\
Z-Z_{s}
\end{array}\right] .
$$

Expand formula (18) to get

$$
x=k^{-1}\left[a_{0}\left(X_{0}-X_{s}\right)+b_{0}\left(Y_{0}-Y_{s}\right)+c_{0}\left(Z_{0}-Z_{s}\right)\right] \text {, }
$$

$$
y=k^{-1}\left[a_{1}\left(X_{0}-X_{s}\right)+b_{1}\left(Y_{0}-Y_{s}\right)+c_{1}\left(Z_{0}-Z_{s}\right)\right] \text {, }
$$

$$
-e=k^{-1}\left[a_{2}\left(X_{0}-X_{s}\right)+b_{2}\left(Y_{0}-Y_{s}\right)+c_{2}\left(Z_{0}-Z_{s}\right)\right] \text {. }
$$

Divide formula (19) to formula (21) by two, and the final expression is as follows:

$$
\begin{aligned}
& x=-e \frac{a_{0}\left(X_{0}-X_{s}\right)+b_{0}\left(Y_{0}-Y_{s}\right)+c_{0}\left(Z_{0}-Z_{s}\right)}{a_{2}\left(X_{0}-X_{s}\right)+b_{2}\left(Y_{0}-Y_{s}\right)+c_{2}\left(Z_{0}-Z_{s}\right)}, \\
& y=-e \frac{a_{1}\left(X_{0}-X_{s}\right)+b_{1}\left(Y_{0}-Y_{s}\right)+c_{1}\left(Z_{0}-Z_{s}\right)}{a_{2}\left(X_{0}-X_{s}\right)+b_{2}\left(Y_{0}-Y_{s}\right)+c_{2}\left(Z_{0}-Z_{s}\right)} .
\end{aligned}
$$

\section{Structural Deformation Monitoring Data Analysis}

4.1. Accuracy Analysis of Close-Range Photogrammetry Equipment. In the experiment, close-range photogrammetric equipment was used to capture the images of the measured objects, and then the accuracy of the equipment was verified by using PhotoModeler Pro5 software. According to the verification results, the coordinates and coordinate accuracy of the five measurement points of the measured objects could be obtained. The results are shown in Table 1 , and the precision unit is $\mathrm{mm}$.

According to the data in Table 1, we can see the coordinate values of the five measuring points measured by the close-range photogrammetry equipment and the accuracy values between the coordinate values. In order to observe the data in the table more intuitively, we convert the data into a graph form, and the final result is shown in Figure 1.

According to the data in Figure 1, we can see that the $X$-axis coordinates of the measurement points taken by the close-range photogrammetric equipment are all positive, indicating that these measurement points are distributed on the right side of the $X$-axis. The data in the figure shows that the accuracy of $X$-axis, $Y$-axis, and $Z$-axis in close-range photogrammetry equipment ranges from 0.00102 to $0.00322 \mathrm{~mm}$, from 0.00107 to $0.00331 \mathrm{~mm}$, and from 0.00110 to $0.00212 \mathrm{~mm}$, respectively. It can be seen that the precision values of all coordinates of the measurement points are less than $0.1 \mathrm{~mm}$, indicating that the
TABLE 1: Measuring point coordinates and accuracy statistics table.

\begin{tabular}{ccccccc}
\hline & $X$ & $Y$ & $Z$ & $X$-precision & $Y$-precision & $Z$-precision \\
\hline 1 & 0.146 & -0.384 & 0.257 & 0.00322 & 0.00331 & 0.00110 \\
2 & 0.279 & 0.681 & -0.334 & 0.00131 & 0.00111 & 0.00141 \\
3 & 0.242 & -0.322 & -0.241 & 0.00312 & 0.00314 & 0.00212 \\
4 & 0.357 & 0.935 & 0.376 & 0.00102 & 0.00107 & 0.00113 \\
5 & 0.196 & 0.472 & 0.164 & 0.00113 & 0.00122 & 0.00211 \\
\hline
\end{tabular}

close-range photogrammetric equipment has a good effect on the calibration of the measurement points.

\subsection{Using Close-Range Photogrammetry to Analyze the Bridge}

4.2.1. Coordinates of Bridge Pier Measuring Point. Measure the $\mathrm{x}, \mathrm{y}$, and $\mathrm{z}$ coordinate values in the space coordinate system using the six-point close-range photogrammetry technology of the bridge pier, then use the direct linear transformation and the collinearity equation to calculate and measure the coordinate transformation space, and obtain $\mathrm{x}, \mathrm{y}, \mathrm{z}$ of the auxiliary space coordinate system. The results are shown in Table 2 .

According to the data in Table 2, we can see the coordinates of the bridge pier measurement points in the space coordinate system, as well as the auxiliary space coordinates calculated by the direct linear transformation and the collinear condition equation. In order to observe the data in the table more intuitively, we convert the data into a graph form, and the final result is shown in Figure 2.

According to the data in Figure 2, we can see that the $x$ axis coordinate range of the bridge pier measurement point in the space coordinate system is $40 \sim 55$, and there are more points in the range of 50 55. The $y$-axis coordinates range from 30 to 55 , and there are many points falling between 40 and 50 . The coordinate range of the $z$-axis is $35-50$, and there are many points that fall between 35 and 40 . The $X$-axis coordinate range of the pier measurement point in the auxiliary space coordinate system is $25 \sim 40$, the $Y$-axis coordinate range is $8 \sim 36$, and the $Z$-axis coordinate range is $10 \sim 35$.

4.2.2. Deformation Coordinates of Bridge Cables. Analyze the cable deformation on the bridge, and calculate the $X$-axis and $Y$-axis spatial coordinates of the six measurement points of the bridge cable under no load, $10 \mathrm{~N}$ and $20 \mathrm{~N}$ loads. The final results are shown in Table 3.

According to the data in Table 3, we can see that the spatial coordinates of the $X$-axis and $Y$-axis of the bridge cables under no load and $10 \mathrm{~N}$ and $20 \mathrm{~N}$ loads have little difference. In order to observe the data changes more intuitively, we convert the data in the table into a graph form, and the final result is shown in Figure 3.

According to the data in Figure 3, bridge cable measurement point coordinates are distributed in the space coordinate system in the fourth quadrant, cable bridge under 


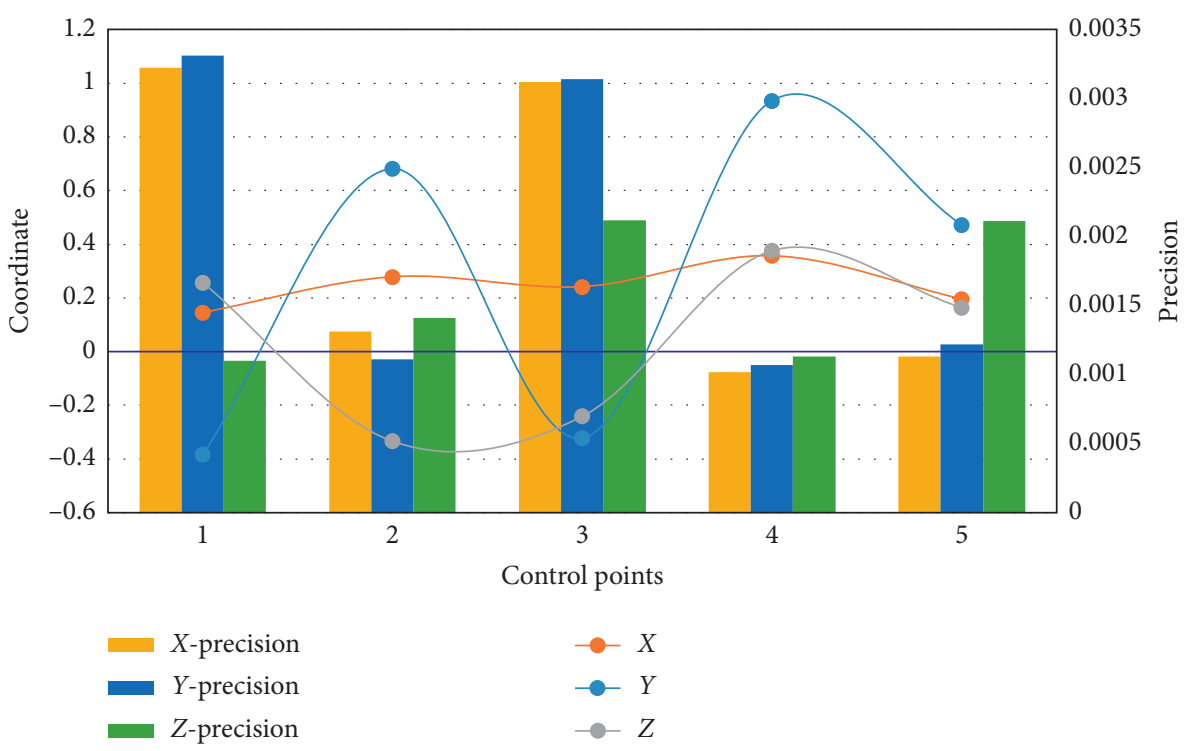

FIGURE 1: Measuring point coordinates and accuracy statistics.

TABLE 2: Object coordinate statistics of bridge pier measurement points.

\begin{tabular}{ccccccc}
\hline & $x$ & $y$ & $z$ & $X$ & $Y$ & $Z$ \\
\hline 1 & 42.96 & 39.66 & 36.22 & 28 & 15 & 11 \\
2 & 54.08 & 44.32 & 37.61 & 39 & 31 & 13 \\
3 & 53.51 & 43.45 & 48.14 & 40 & 28 & 35 \\
4 & 53.09 & 33.97 & 47.31 & 37 & 8 & 33 \\
5 & 51.28 & 45.21 & 35.82 & 35 & 33 & 10 \\
6 & 46.25 & 52.44 & 37.46 & 34 & 36 & 15 \\
\hline
\end{tabular}

no-load measurement point $X$-axis coordinate range between 40 and 50 and maximum and minimum values are 49.03 and 42.36 , respectively, and $\mathrm{Y}$ coordinates range between -4 and -8 and maximum and minimum values of 4.92 and 7.79 , respectively. The $X$-axis coordinate range of the bridge cable under $10 \mathrm{~N}$ and $20 \mathrm{~N}$ loads is consistent with that under no load. The $X$-axis maximum and minimum values of the measuring points under $10 \mathrm{~N}$ loads are 49.06 and 42.38, respectively, and the $Y$-axis maximum and minimum values are -4.81 and -7.76 , respectively. Under the action of $20 \mathrm{~N}$ load, the maximum and minimum values of $X$-axis coordinates of the measurement point are 49.01 and 42.33, respectively, and the maximum and minimum values of $Y$-axis are -4.77 and -7.74 , respectively. It can be seen that the spatial coordinates of bridge cable deformation have little difference, and the coordinates of measuring points have little change.

4.2.3. Analysis of Accuracy of Close-Range Photogrammetry. In order to verify the accuracy of the close-range photogrammetry technique, two methods are used to compare the measurement results. The calculation results of the bridge cables under the $10 \mathrm{~N}$ and $20 \mathrm{~N}$ loads and the measurement results obtained by the close-range photogrammetry technique are statistically calculated, and the error values are calculated. The final results are shown in Table 4.
According to the data in Table 4, we can see that the error generated when the load is $10 \mathrm{~N}$ is larger than the error generated when the load is $20 \mathrm{~N}$. In order to observe the data in the table more intuitively, we convert the data in the table into a graph form, and the final result is shown in Figure 4.

According to the data in Figure 4, we can see that under the condition of a load of $10 \mathrm{~N}$, the close-range photogrammetry technique has a maximum error of position 1, with an error value of $5.69 \%$, and a minimum error of position 4 , with an error value of $2.36 \%$. When the load is 20 $\mathrm{N}$, the maximum error is No. 1 position, the error value is $1.86 \%$, the minimum error is No. 6 position, and the error value is $0.74 \%$. This can be seen from the data in the figure that the error generated when the load of each measuring point is $10 \mathrm{~N}$ is greater than the error generated when the load is $20 \mathrm{~N}$.

4.2.4. Error Analysis of Measuring Points. The measurement errors of the six measurement points on $X$-axis, $Y$-axis, $Z$ axis, and the direction of point position were analyzed, and the accuracy values of each measurement point on $X$ axis, $Y$-axis, $Z$-axis, and the direction of point position were calculated. The statistical results were shown in Table 5 (unit: $\mathrm{mm}$ ).

According to the data in Table 5, we can see the error values of the six measurement points on the $X$-axis, $Y$-axis, $Z$-axis, and the point direction. According to the data in the table, the error of the measurement points can be analyzed. In order to observe the data in the table more intuitively, we convert the data in the table into a graph form, and the final result is shown in Figure 5.

According to the data in Figure 5, we can see that the largest measurement error of these six measurement points in the $X$-axis direction is point 5 , and the error value is $1.135 \mathrm{~mm}$. The largest measurement error in the $Y$-axis direction is point 1 , with an error value of $1.879 \mathrm{~mm}$. The 


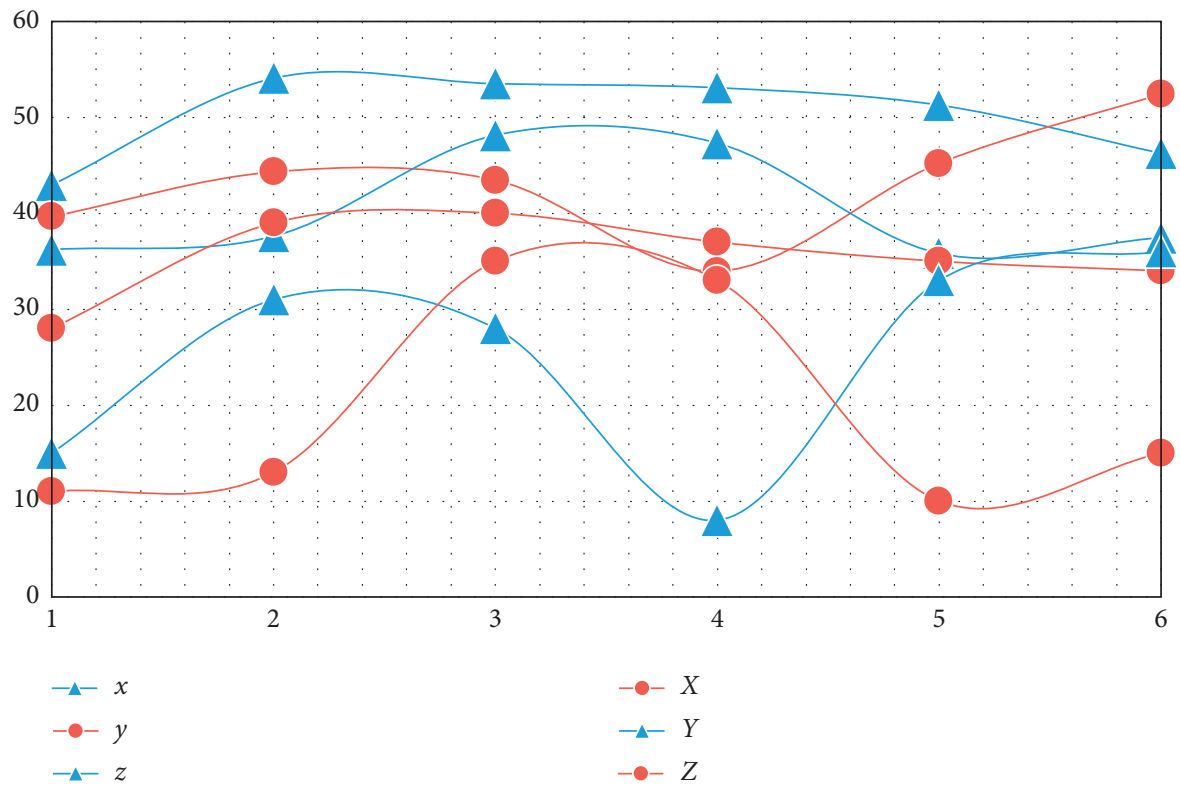

FIGURE 2: The coordinate statistics of the bridge pier measurement points.

TABle 3: Cable deformation coordinate statistics.

\begin{tabular}{|c|c|c|c|c|c|c|}
\hline & No load- $X$ & No load- $Y$ & $10 \mathrm{~N}-X$ & $10 \mathrm{~N}-Y$ & $20 \mathrm{~N}-X$ & $20 \mathrm{~N}-Y$ \\
\hline 1 & 44.39 & -4.92 & 44.37 & -4.81 & 44.41 & -4.77 \\
\hline 2 & 43.38 & -5.18 & 43.41 & -5.22 & 43.36 & -5.16 \\
\hline 3 & 49.03 & -6.04 & 49.06 & -6.11 & 49.01 & -6.08 \\
\hline 4 & 45.56 & -6.16 & 45.53 & -6.13 & 45.59 & -6.1 \\
\hline 5 & 47.63 & -7.18 & 47.66 & -7.21 & 47.62 & -7.16 \\
\hline 6 & 42.36 & -7.79 & 42.38 & -7.76 & 42.33 & -7.74 \\
\hline
\end{tabular}

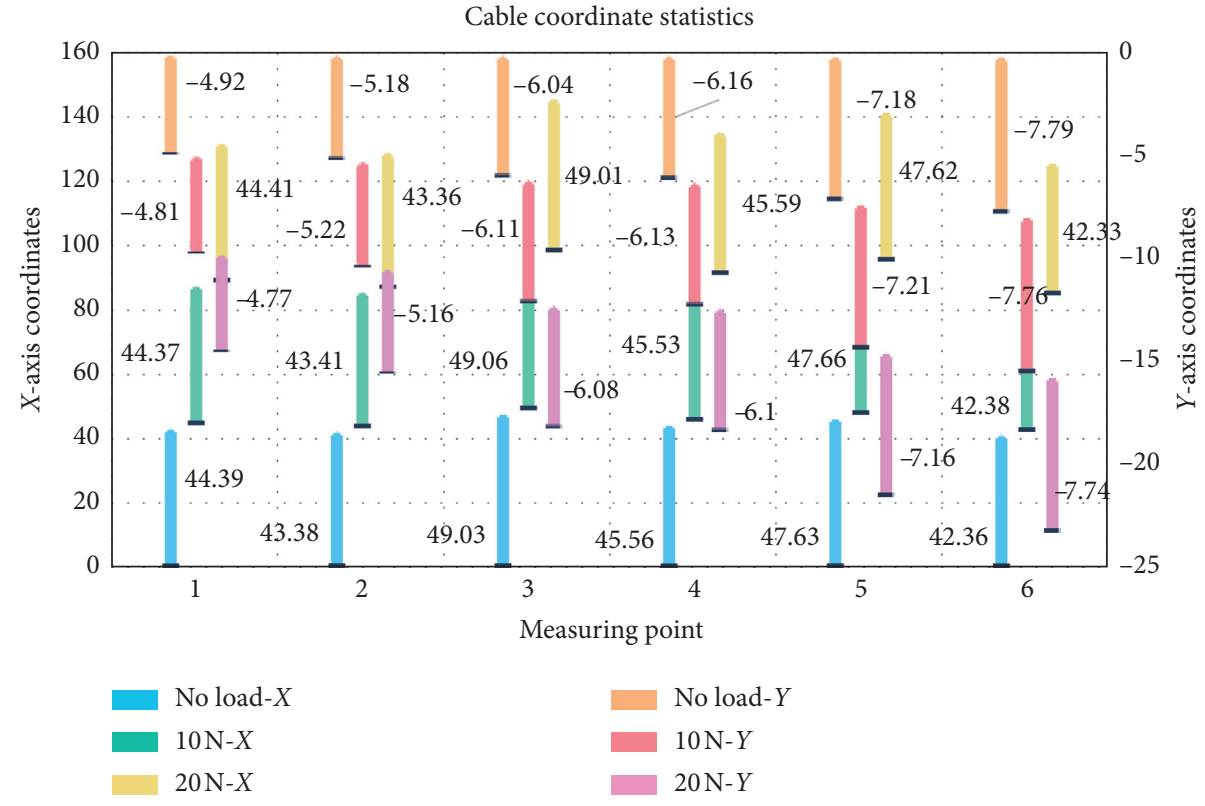

FIgURE 3: Coordinate statistics of bridge cable deformation. 
TABle 4: Comparison of cable deformation.

\begin{tabular}{|c|c|c|c|c|c|c|}
\hline & Calculated-10 N & Measurements-10 N & Error-10 N (\%) & Calculated-20 N & Measurements- $20 \mathrm{~N}$ & Error-20 N (\%) \\
\hline 1 & 1.23 & 1.3 & 5.69 & 2.15 & 2.19 & 1.86 \\
\hline 2 & 1.12 & 1.07 & 4.46 & 2.42 & 2.4 & 0.83 \\
\hline 3 & 1.19 & 1.1 & 7.56 & 2.23 & 2.26 & 1.35 \\
\hline 4 & 1.27 & 1.3 & 2.36 & 2.82 & 2.78 & 1.42 \\
\hline 5 & 1.03 & 1.0 & 2.91 & 2.58 & 2.61 & 1.16 \\
\hline 6 & 1.34 & 1.3 & 2.99 & 2.71 & 2.69 & 0.74 \\
\hline
\end{tabular}

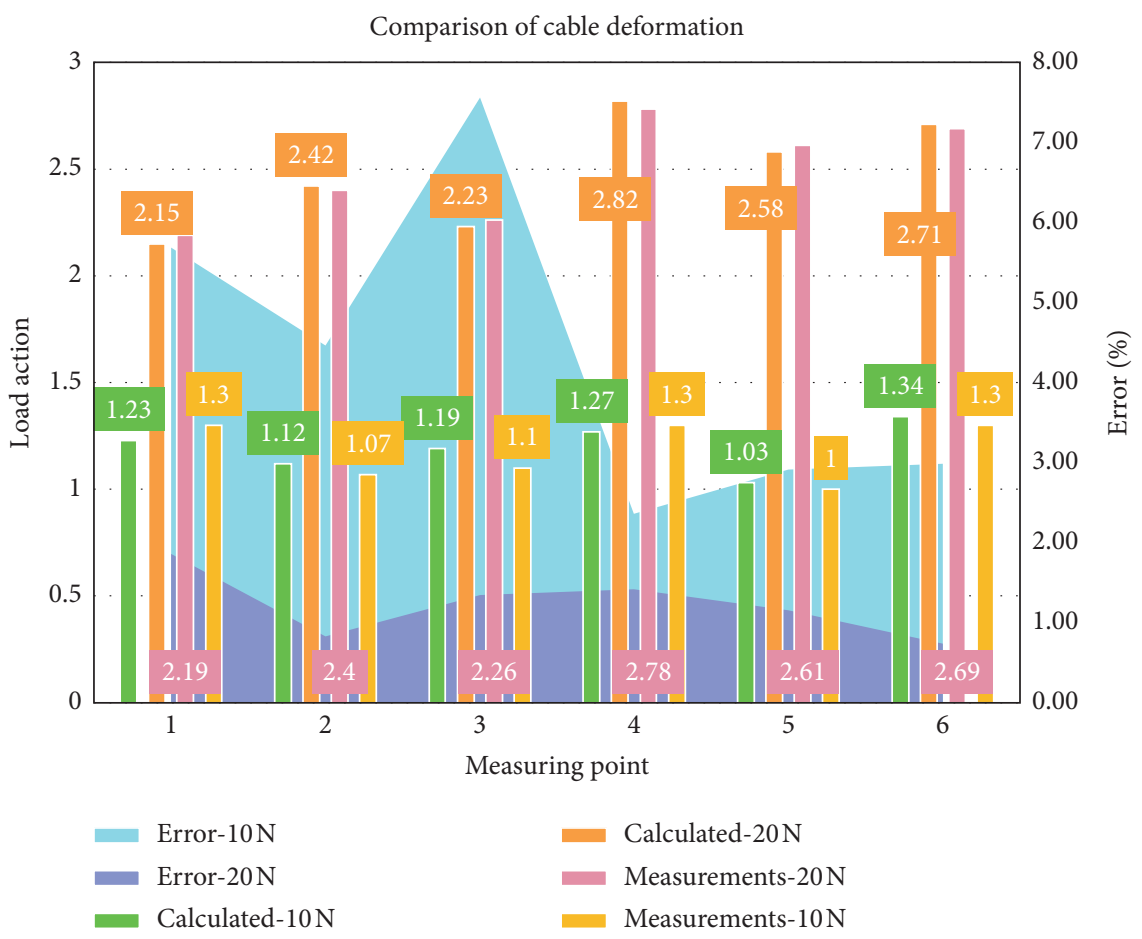

Figure 4: Comparison of deformation of bridge cables.

TABle 5: Accuracy statistics of measuring points.

\begin{tabular}{ccccc}
\hline & $X$ & $Y$ & $Z$ & Point position \\
\hline 1 & 0.539 & 1.879 & 0.527 & 1.623 \\
2 & 0.655 & 1.759 & 0.487 & 1.952 \\
3 & 0.342 & 1.412 & 0.478 & 1.369 \\
4 & 0.829 & 1.543 & 0.277 & 1.293 \\
5 & 1.135 & 1.621 & 0.661 & 1.082 \\
6 & 0.257 & 1.733 & 0.515 & 1.334 \\
\hline
\end{tabular}

largest measurement error in the $Z$-axis direction is point 5 , and the error value is $0.661 \mathrm{~mm}$. The point with the largest measurement error is point 2, with an error value of $1.952 \mathrm{~mm}$. It can be seen that the coordinate error of the measuring point in this experiment is not big, and the measuring instrument used is more accurate.

4.3. Comparison of Measurement Performance of Different Methods. Under the same conditions, total station method, direct linear transformation method, and other photogrammetric methods were used to calculate and analyze the data of measurement points. By comparing with the actual values, the advantages of the measurement and calculation method selected in this paper were analyzed. The calculation results are shown in Table 6 .

According to the data in Table 6, we can see that the measurement calculation method with the smallest difference from the actual value is the direct linear transformation method, and the other methods have more errors. In order to observe the data in the table more intuitively, we convert the data in the table into a graph form, and the final result is shown in Figure 6.

According to the data in Figure 6, we can see that the results obtained by the total station measurement and calculation method are reduced by about $0.004 \sim 0.007 \mathrm{~mm}$ compared with the actual value; the results obtained by the direct linear transformation method are increased by about 0.002 0.004 $\mathrm{mm}$ compared with the actual value; and the results obtained by other measurement and calculation methods are increased by about $0.004 \sim 0.006 \mathrm{~mm}$ compared with the actual value. Thus, the difference between the results obtained by the direct linear transformation calculation 


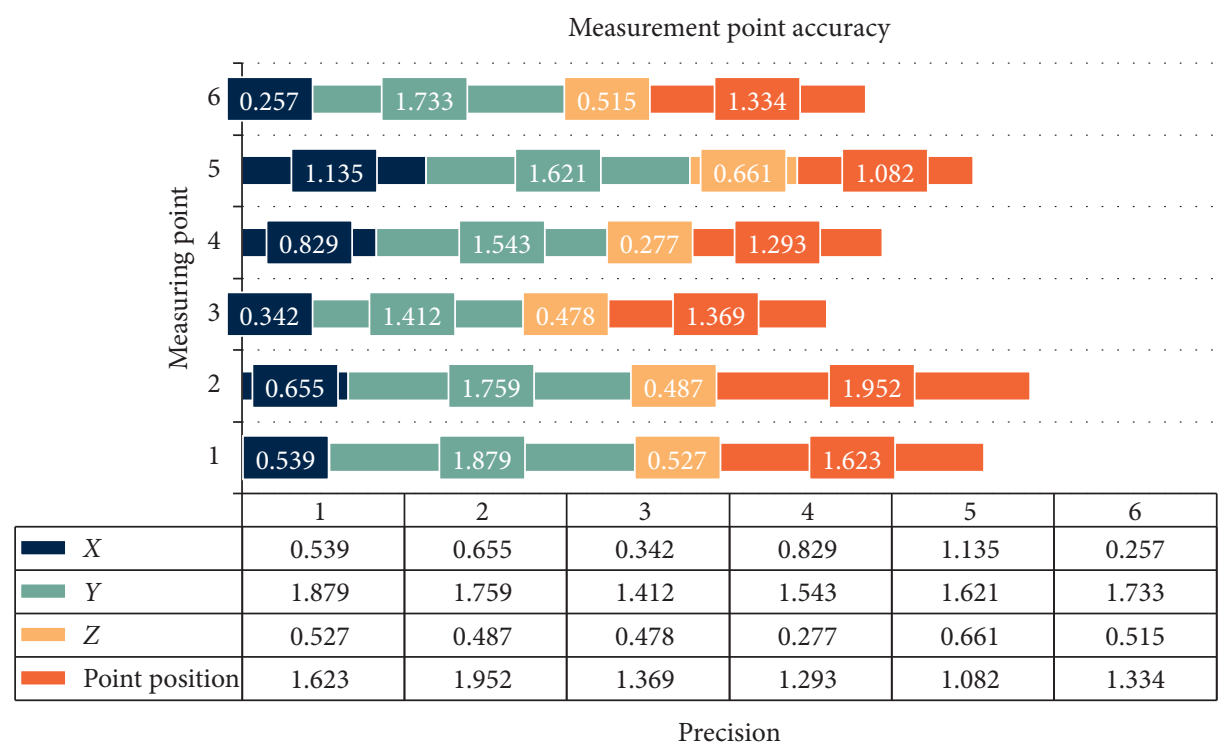

FIGURE 5: Accuracy statistics of measurement points.

TABLE 6: Comparison table of calculation results of different methods.

\begin{tabular}{lcccc}
\hline & Actual value & Total station & DLT & Others \\
\hline 1 & 1.322 & 1.315 & 1.326 & 1.328 \\
2 & 1.641 & 1.637 & 1.644 & 1.649 \\
3 & 1.417 & 1.412 & 1.419 & 1.421 \\
4 & 2.364 & 2.358 & 2.366 & 2.368 \\
5 & 1.846 & 1.841 & 1.848 & 1.850 \\
6 & 2.231 & 2.224 & 2.235 & 2.237 \\
\hline
\end{tabular}

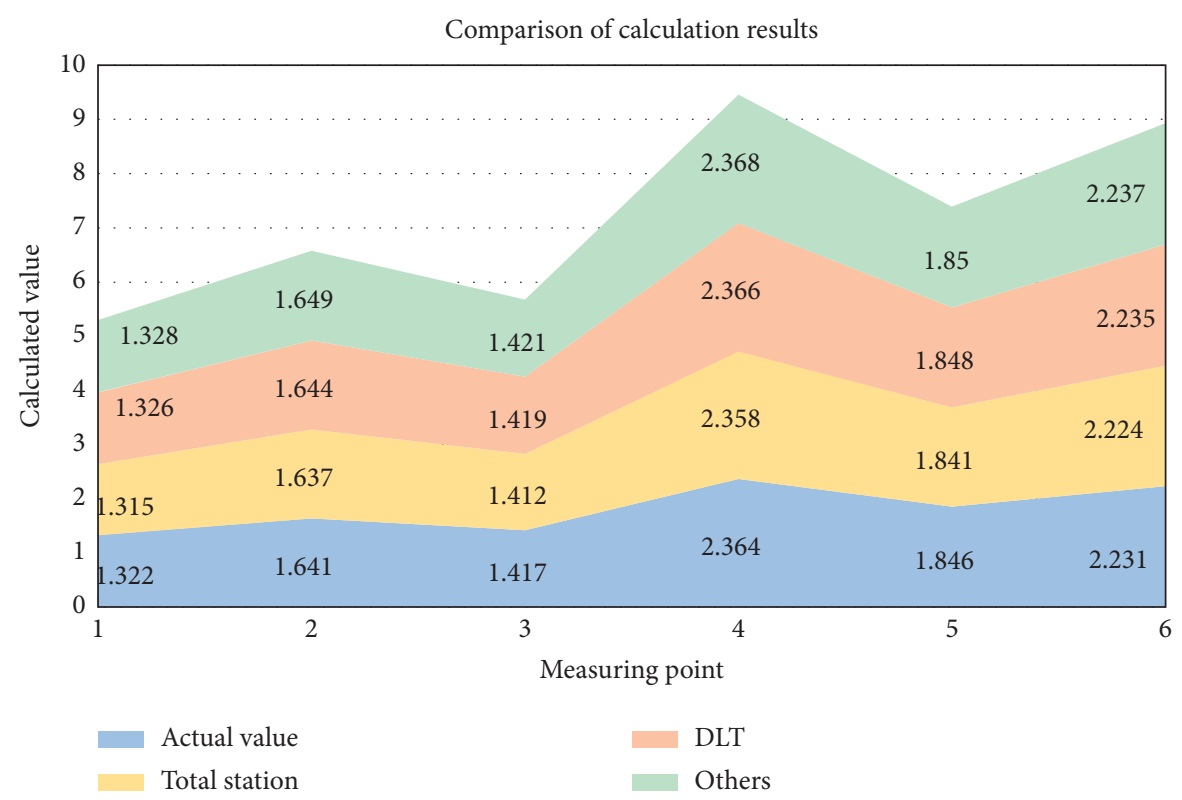

FIgURE 6: Comparison of calculation results of different methods. 
method and the actual value is the smallest, indicating that the method used in this paper has a better performance in calculation and measurement.

\section{Conclusions}

The problem of bridge deformation monitoring has always been the focus of scholars' research in architecture and other fields. Bridges are a very important part of transportation, and they are also one of the important hubs connecting the economic exchanges of various regions. Research on the problem of bridge deformation monitoring can effectively prevent the occurrence of bridge safety accidents, eliminate the threat to people's lives and property safety, and play an important role in the future bridge design and bridge maintenance.

In this study, structural deformation monitoring based on close-range photogrammetry was studied. The coordinates of bridge pier and cable deformation were obtained using close-range photogrammetry, and the obtained data were calculated using direct linear transformation and collinear equation. The deformation of bridge measurement points is analyzed experimentally, the error and accuracy of close-range photogrammetry are analyzed, and the accuracy of calculation results between different measurement and calculation methods is compared.

The final results of this study show that the coordinates of measuring points of the bridge cable are distributed in the fourth quarter of the spatial coordinate system. Under no load, the $X$-axis coordinates of measuring points of the bridge cable range from 40 to 50 , with the maximum and minimum values of 49.03 and 42.36, respectively, and the $Y$ axis coordinates range from -8 to -4 , with the maximum and minimum values of -4.92 and -7.79 , respectively. In the close-range measurement technology, the error generated when the load action of each measurement point is $10 \mathrm{~N}$ is larger than that generated when the load action is $20 \mathrm{~N}$. In this experiment, the coordinate error of the measurement point is not large, and the measurement instrument used is of high accuracy. In addition, the results obtained by the direct linear transformation calculation method have the smallest difference from the actual values in different measurement calculation methods and have better calculation and measurement performance.

\section{Data Availability}

The data underlying the results presented in the study are available within the manuscript.

\section{Conflicts of Interest}

The authors declare no conflicts of interest.

\section{Authors' Contributions}

All authors read and approved the final version of the manuscript.

\section{Acknowledgments}

This work was supported by the Open Fund of the State Key Laboratory of Mountain Bridge and Tunnel Engineering (SKLBT-19-005).

\section{References}

[1] Z. Lv, X. Li, H. Lv, and W. Xiu, "BIM big data storage in WebVRGIS," IEEE Transactions on Industrial Informatics, vol. 16, no. 4, pp. 2566-2573, 2016.

[2] H. G. Kim and H. S. Yun, "Shape deformation monitoring for VLBI antenna using close-range photogrammetry and total least squares," Journal of the Korean Society of Surveying, Geodesy, Photogrammetry and Cartography, vol. 34, no. 1, pp. 99-107, 2016.

[3] T. Yunchao, F. Wanhui, and F. Wenxian, "Compressive properties of rubber-modified recycled aggregate concrete subjected to elevated temperatures," Construction and Building Materials, vol. 268, Article ID. 121181, 2020.

[4] B. Chen, T. Hu, Z. Huang et al., "A spatio-temporal clustering and diagnosis method for concrete arch dams using deformation monitoring data," Structural Health Monitoring, vol. 18, no. 6, pp. 1355-1371, 2019.

[5] P. Lopato and M. Herbko, "Microwave structural health monitoring sensor for deformation measurement of bended steel structures: influence of curvature effect," Radioengineering, vol. 26, no. 4, pp. 1060-1066, 2017.

[6] G. Sun, "Structural deformation monitoring of flight vehicles based on optical fiber sensing technology: a review and outlook," Hangkong Xuebao/Acta Aeronautica et Astronautica Sinica, vol. 20, no. 20, pp. 1-21, 2020.

[7] E. L. Sachi Nandan Mohanty Lydia, M. Elhoseny, M. Majid, G. Al Otaibi, and K. Shankar, "Deep learning with LSTM based distributed data mining model for energy efficient wireless sensor networks," Physical Communication, vol. 40, Article ID. 101097, 2020.

[8] J. Zhang, Y. Tian, C. Yang et al., "Vibration and deformation monitoring of a long-span rigid-frame bridge with distributed long-gauge sensors," Journal of Aerospace Engineering, vol. 30, no. 2, pp. 1-9, 2017.

[9] M. Pepe and D. Costantino, "Techniques, tools, platforms and algorithms in close range photogrammetry in building $3 \mathrm{D}$ model and 2D representation of objects and complex architectures," Computer-Aided Design and Applications, vol. 18, no. 1, pp. 42-65, 2020.

[10] I. Shufrin, E. Pasternak, and A. V. Dyskin, "Deformation analysis of reinforced-core auxetic assemblies by close-range photogrammetry," Physica Status Solidi (B), vol. 253, no. 7, pp. 1342-1358, 2016.

[11] H. Zhang, S. Qu, H. Li, J. Luo, and W. Xu, "A moving shadow elimination method based on fusion of multi-feature," IEEE Access, vol. 8, pp. 63971-63982, 2020.

[12] K. Geetha, V. Anitha, M. Elhoseny, S. Kathiresan, P. Shamsolmoali, and M. M. Selim, "An evolutionary lion optimization algorithm-based image compression technique for biomedical applications," Expert Systems, vol. 38, no. 2, 2020.

[13] M. B. Campos, A. M. G. Tommaselli, L. F. Castanheiro, R. A. Oliveira, and E. Honkavaara, "A fisheye image matching method boosted by recursive search space for close range photogrammetry," Remote Sensing, vol. 11, no. 12, pp. 1404-1405, 2019. 
[14] J. erňava, F. Chud, T. Daniel et al., "The vertical accuracy of digital terrain models derived from the close-range photogrammetry point cloud using different methods of interpolation and resolutions," Central European Forestry Journal, vol. 66, no. 4, pp. 198-205, 2019.

[15] H. E. D. Fawzy and E. S. E. Din, "Monitoring and assessment OF road cracks and potholes using digital close range photogrammetry $3 \mathrm{D}$ reconstruction technique," International Journal of Civil Engineering and Technology, vol. 11, no. 1, pp. 119-128, 2020.

[16] A. B. Ebrahim, "Slab diflection analysis using digital closerange photogrammetry," International Journal of Ence and Research (IJSR), vol. 8, no. 1, pp. 1006-1010, 2019.

[17] E. Ata and A. Pirti, "Monitoring the structural deformation of davutpasa barrack by using geodetic methods," Tehnicki Vjesnik, vol. 25, no. 3, pp. 944-947, 2018.

[18] M. Azimi, A. Rasoulnia, Z. Lin et al., "Improved semi-active control algorithm for hydraulic damper-based braced buildings," Structural Control \& Health Monitoring, vol. 24, no. 11, pp. 1-17, 2017.

[19] A. F. H. Al-Maliki, N. M. Faleh, and A. A. Alasadi, "Finite element formulation and vibration of nonlocal refined metal foam beams with symmetric and non-symmetric porosities," Structural Monitoring and Maintenance, vol. 06, no. 2, pp. 147-159, 2019.

[20] J. S. Lew, "Parameter variations of identified model for static deformation analysis of arch dam," Civil Engineering and Architecture, vol. 010, no. 008, pp. 894-900, 2016.

[21] Y. Fujino and Y. Kawai, "Technical developments in structural engineering with emphasis on steel bridges in Japan," Journal of Jsce, vol. 4, no. 1, pp. 211-226, 2016.

[22] A. Ellenberg, A. Kontsos, F. Moon, and I. Bartoli, "Bridge related damage quantification using unmanned aerial vehicle imagery," Structural Control and Health Monitoring, vol. 23, no. 9, pp. 1168-1179, 2016.

[23] J. Fang, K. Wang, and Y. Huang, "Weld pool image recognition of humping formation process in high speed GMAW," Hanjie Xuebao/Transactions of the China Welding Institution, vol. 40, no. 2, pp. 42-46, 2019.

[24] N. Nitta, K. Nakamura, and N. Babaguchi, "Constructing geospatial concept graphs from tagged images for geo-aware fine-grained image recognition," ISPRS International Journal of Geo-Information, vol. 9, no. 6, pp. 354-355, 2020.

[25] V. Vyalov, A. Y. Andreev, and A. Andreev, "Convolutional neural networks for optical image recognition of ships," Transactions of the Krylov State Research Centre, vol. 3, no. 393, pp. 91-96, 2020.

[26] Y. Tang and M. Elhoseny, "Computer network security evaluation simulation model based on neural network," Journal of Intelligent \& Fuzzy Systems, vol. 37, no. 3, p. 3197, 2019.

[27] S.-J. Jeong, I.-K. Hwang, I.-S. Cho, and H.-S. Kim, "Estimation of chemical composition of Al-Si cast alloys using image recognition," Korean Journal of Metals and Materials, vol. 57, no. 3, pp. 184-192, 2019.

[28] R. Mrginean, A. Andreica, L. Dioan et al., "Butterfly effect in chaotic image segmentation," Entropy, vol. 22, no. 9, pp. 1028-1029, 2020.

[29] M. Ali, R. F. Chisab, and F. M. Lahmood, "Mammogram image segmentation for improving the diagnosis of dense breast issues," International Journal of Engineering and Technology, vol. 8, no. 1, pp. 44-52, 2019.

[30] R. Sahoo and C. Sekhar, "Detection of diabetic retinopathy from retinal fundus image using wavelet based image segmentation," International Journal of Computer Applications, vol. 182, no. 47, pp. 46-50, 2019.

[31] K. Ghathwan and A. J. Mohammed, "Intelligent bio-inspired whale optimization algorithm for color image based segmentation," Pertanika Journal of Ence and Technology, vol. 28, no. 4, pp. 1389-1411, 2020.

[32] Q. Wang and P. Lu, "Research on application of artificial intelligence in computer network technology," International Journal of Pattern Recognition and Artificial Intelligence, vol. 33, no. 05, p. 1959015, 2019.

[33] B. Zhu, S. Ma, R. Xie, J. Chevallier, and Y.-M. Wei, "Hilbert spectra and empirical mode decomposition: a multiscale event analysis method to detect the impact of economic crises on the European carbon market," Computational Economics, vol. 52, no. 1, pp. 105-121, 2018. 